\title{
ON LEFT THICKNESS OF SUBSETS IN SEMIGROUPS
}

JAMES C. S. WONG ${ }^{1}$

\begin{abstract}
ABSTRACr. We reformulate the concept of left thickness in a semigroup introduced by $\mathrm{H}$. Junghenn in Amenability of function spaces on thick subsemigroups, Proc. Amer. Math. Soc. 75 (1979), 37-41, and obtain a number of interesting new characterisations of left thickness without assuming left amenability. Moreover, these characterisations are similar in nature to familiar characterisations of left amenability on semigroups. We also sharpen some of Junghenn's results, paving the way for extension to locally compact semigroups.
\end{abstract}

1. Introduction. Let $S$ be a semigroup and $T$ a subset of $S$. $T$ is said to be left thick in $S$ if for each finite subset $\sigma$ in $S$, there is some $s \in S$ such that $\sigma s \subset T$. In 1965, T. Mitchell [11] showed that if $S$ is left amenable, then $T$ is left thick in $S$ if and only if there is some left invariant mean $m$ on the bounded functions $B(S)$ on $S$ such that $m\left(\xi_{T}\right)=1$ where $\xi_{T}$ is the characteristic functions of $T$. Recently, H. Junghenn [9] extended this concept to left $F$-thickness (or $F$-left thickness) with respect to a left introverted left invariant norm closed subalgebra $F$ of $B(S)$ and obtained similar characterisations of left $F$-thickness without assuming a priori left amenability of $F$. In this paper, we shall reformulate Junghenn's definition of left $F$-thickness and obtain a number of interesting new characterisations, again without the left amenability assumption. On the other hand, these characterisations (e.g. the Dixmier's Criterion and Localisation Theorem, see $\$ 3$ below) closely resemble some well-known characterisations of left amenability on semigroups. We also sharpen some of the results in Junghenn [9]. In particular we show that $T$ is left thick in $S$ if and only if the closure of $T$ is left thick in the closure of $S$, where the closure is taken in some suitable topology.

2. Terminologies. For terminologies in analysis on semigroups, we shall follow Junghenn [9] except that the characteristic function of $T$ is denoted by $\xi_{T}$ and not by $\chi_{T}$ (which stands for the characteristic functional of $T$; see Wong [16]). Let $S$ be a semigroup and $F$ a left invariant linear sublattice of the bounded functions $B(S)$ containing the constants. However, unlike in Junghenn [9], $F$ need not be a left introverted norm closed subalgebra (which is necessarily a sublattice). A subset $T$ in

Received by the editors April 14, 1981.

AMS (MOS) subject classifications (1970). Primary 43A07.

Key words and phrases. Semigroups, left thick subsets, means, bounded functions on semigroups.

'Research supported by Natural Sciences and Engineering Research Council of Canada Grant No. A8227. 
$S$ is called left $F$-thick (or $F$-left thick, as in [9]) if for each $\varepsilon>0$, each $g \in F(T)=$ $\left\{f \in F: \xi_{T} \leqslant f \leqslant 1\right\}$ and each finite subset $\sigma=\left\{s_{1}, s_{2}, \ldots, s_{k}\right\} \subset S$, there is some $s \in S$ such that $g\left(s_{i} s\right)>1-\varepsilon$ for all $1 \leqslant i \leqslant k$. Also, define $e(s) f=f(s), m_{l}(f)(s)$ $=m(L(s) f), s \in S, f \in F, m \in F^{*}, L(s) f(t)=f(s t), t \in S$. Let $\mathrm{Cl} e(T)$ denote the weak* (i.e. $\sigma\left(F^{*}, F\right)$ ) closure of $e(T)$ in $F^{*}$ and let $M(F)$ be the set of all means on $F$.

3. Main results. We first improve upon some of Junghenn's results in [9].

THEOREM 3.1. Let $T$ be a subset in $S$. Then the following four statements on $T$ are equivalent.

(a) $T$ is left F-thick.

(b) For any $\varepsilon>0$, any finite subset $D$ of $F(T)$ and any finite subset $\left\{s_{1}, s_{2}, \ldots, s_{k}\right\}$ of $S$, there is some $s \in S$ such that for each $g \in D, k^{-1} \Sigma\left\{g\left(s_{i} s\right): 1<i \leqslant k\right\}>1-\varepsilon$.

(c) There is some $\lambda \in \mathrm{Cl} e(S)$ such that $\lambda(L(s) g)=1$ for all $s \in S, g \in F(T)$.

(d) There is some $\mu \in M(F)$ such that $\mu(L(s) g)=1$ for all $s \in S, g \in F(T)$.

Proof. The proofs of the implications $(a) \Rightarrow(b)$ and $(c) \Rightarrow(d)$ are as in [9, Theorem 1]. For (b) $\Rightarrow$ (c), we also can follow [9, Theorem 1, (b) $\Rightarrow$ (c)] except that we have to replace the expression $e(s) \lambda(g)$ by $\lambda(L(s) g)$ since the product $e(s) \lambda$ is not yet defined. (Note that there is a misprint in [9]: " $M(F)$ " should read " $\mathrm{Cl} e(S)$ ".) For (d) $\Rightarrow(\mathrm{a})$, suppose (a) is not true, then as in [9], there is some $h \in F(T), \varepsilon>0,\left\{s_{1}, s_{2}, \ldots, s_{k}\right\} \subset S$ such that for each $s \in S, h\left(s_{i} s\right) \leqslant 1-\varepsilon$ for some $i$. Consider the function $g=k^{-1} \Sigma\left\{L\left(s_{i}\right) h: 1 \leqslant i \leqslant k\right\} \in F, g(s)=$ $k^{-1} \Sigma\left\{h\left(s_{i} s\right): 1 \leqslant i \leqslant k\right\} \leqslant 1-k^{-1} \varepsilon$ for any $s \in S$. Hence if (d) holds, we have $1=k^{-1} \sum\left\{\mu\left(L\left(s_{i}\right) h\right): 1 \leqslant i \leqslant k\right\}=\mu(g) \leqslant 1-k^{-1} \varepsilon$ which is a contradiction. Hence (a), (b), (c) and (d) are equivalent.

THEOREM 3.2. If $F$ is left introverted, i.e. $m_{l}(F) \subset F$ for all $m \in M(F)$, then the four statements(a), (b), (c) and (d) above are all equivalent to

(e) there is some $\mu \in M(F)$ such that $\nu \mu(g)=1$ for all $\nu \in M(F), g \in F(T)$, where $\nu \mu=\nu \odot \mu$ is the Arens product in $F^{*}$ with $\nu \odot \mu(f)=\nu(\mu,(f)), f \in F$.

Proof. As in [9]. Note that left introvertedness ensures that the Arens product is well defined.

THEOREM 3.3. If $F$ is a multiplicative left introverted (i.e. $m_{l}(F) \subset F$ for any multiplicative mean $m$ on $F$ ) norm closed subalgebra of $B(S)$, then the four statements (a), (b), (c) and (d) are all equivalent to both

(f) $\mathrm{Cl} e(T)$ contains a left ideal of $\mathrm{Cl} e(S)$.

(g) $\mathrm{Cl} e(T)$ is left $B\left(S_{1}\right)$-thick in $S_{1}=\mathrm{Cl} e(S)$.

Proof. It is well known that if $F$ is a norm closed subalgebra of $B(S)$, then $\mathrm{Cl} e(S)$ is precisely the set of all multiplicative means on $F$ (Mitchell [12, pp. 119-120]) which is a semigroup under Arens product if $F$ is multiplicative left introverted. Hence (c) $\Rightarrow$ (f) as in [9]. Obviously (f) $\Rightarrow(\mathrm{g})$ (since any left ideal is left $F$-thick for whatever $F)$. Finally assume that $\mathrm{Cl} e(T)$ is left $B\left(S_{1}\right)$-thick in $S_{1}$. Then by Theorem 3.1, applied to $S_{1}$, there is a multiplicative mean $\theta$ on $B\left(S_{1}\right)$ such that 
$\theta(L(m) h)=1$ for all $m \in S_{1}, h \in B\left(S_{1}\right)$ such that $\xi_{\mathrm{Cl} e(T)} \leqslant h \leqslant 1$. Consider the

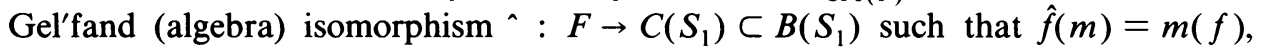
$f \in F, m \in S_{1}$ where $S_{1}$ has the weak* topology $\sigma\left(F^{*}, F\right)$. It is easy to show that ${ }^{\wedge}$ is order preserving, $\hat{1}=1$ and $(L(s) f) \hat{=}=L(e(s)) \hat{f}$ for any $s \in S, f \in F$. Therefore $\mu={ }^{*} \theta$ is a multiplicative mean on $F$. Now let $f \in F(T)$. Then $\xi_{\mathrm{Cl} e(T)} \leqslant \hat{f} \leqslant 1$ since $m(f)=1$ whenever $m \in \mathrm{Cl} e(T)$ and $f \in F(T)$. Hence

$$
\mu(L(s) f) \hat{=}=*(L(s) f)=\theta(L(s) f)^{\hat{n}}=\theta(L(e(s)) \hat{f})=1 \quad \text { for any } s \in S .
$$

Therefore $T$ is left $F$-thick in $S$ and (g) implies (a) which in turn implies (c) by Theorem 3.1.

REMARKS. (1) The above theorems clearly strengthen the result in Junghenn [9, Theorem 1]. Indeed, Theorem 3.1 does not require $F$ to be a subalgebra of $B(S)$. This opens up possible extensions to topological left thickness of subsets in a locally compact semigroup $S$ with respect to topological left invariant linear subspaces of $M(S)^{*}$ (the adjoint of the measure algebra $M(S)$ ) which is not an algebra under pointwise operations. This will be dealt with in another forthcoming paper.

(2) The characterisation (g) is new. In fact Theorem 3.3 remains valid if (g) is replaced by $\left(\mathrm{g}_{1}\right): \mathrm{Cl} e(T)$ is left $W$-thick in $S_{1}$ where $W$ is any left invariant norm closed subalgebra of $B\left(S_{1}\right)$ containing $F=C\left(S_{1}\right)$. The same proof will suffice. In particular, one can take $W=W_{F}$, the norm closed left invariant subalgebra of $B\left(S_{1}\right)$ generated by $C\left(S_{1}\right)$. Note that in general $W_{F}$ may contain non continuous functions on $S_{1}$, since $S_{1}$, though weak* compact, is not a topological semigroup because multiplication in $S_{1}$ is not in general separately continuous in the weak* topology. (As a result, $C\left(S_{1}\right)$ may not even be left translation invariant!) However, if $F=W(S)$, the weakly almost periodic functions on $S$, then $S_{1}$ is a compact (separately continuous) semigroup by Kharaghani [10]. In this case $W_{F}=C\left(S_{1}\right)$ and left $F$-thickness of $T$ is equivalent to left $C\left(S_{1}\right)$-thickness of $\mathrm{Cl} e(T)$. (Or indeed of $e(T)$, see [9, Remark 4, p. 39].)

(3) Theorem 3.3 also says that, for general $T$ and $F, \mathrm{Cl} e(T)$ is left $B\left(S_{1}\right)$-thick in $S_{1}$ if and only if $\mathrm{Cl} e(T)$ contains a left ideal of $S_{1}$. For $F=W(S)$, this follows also from Junghenn [9, Remark 7, p. 39]. In general, a left thick subset need not contain a left ideal.

Next, we reformulate the concept of left thickness and obtain the Localisation Theorem which is used to show that left thickness of $T$ is equivalent to $T$ being right stationary. By definition, $T$ is said to be right $F$-stationary if for each $g \in F$, $0 \leqslant g \leqslant \xi_{T^{\prime}}$, there is a net $s_{\alpha}$ in $S$ such that $R\left(s_{\alpha}\right) g \rightarrow 0$ pointwise on $S$ or equivalently weak* in $B(S)=l_{1}(S)^{*}$. (See Mitchell [11, Lemma 3].) Here $R(s) g$ is the right translate of $g$ by $s$.

THEOREM 3.4. Let $F$ be a lattice (e.g. when $F$ is a norm closed subalgebra of $B(S)$ ). Then the following statements are equivalent:

(a) $T$ is left $F$-thick.

(b) There is a mean $\lambda \in \mathrm{Cl} e(S)$ such that $\lambda(L(s) g)=0$ for all $s \in S$ and all $g \in F$ with $0 \leqslant g \leqslant \xi_{T^{\prime}}$. (Reformulation)

(c) For each $g \in F$ with $0 \leqslant g \leqslant \xi_{T^{\prime}}$, there is a mean $\lambda_{g} \in \mathrm{Cl} e(S)$ such that $\lambda_{g}(L(s) g)=0$ for all $s \in S$. (Localisation) 
(d) $T$ is right $F$-stationary. (Mitchell's Condition)

In addtion, if $F$ is also a multiplicative left introverted norm closed subalgebra of $B(S)$, then these four statements are all equivalent to both:

(e) There is a mean $\lambda \in \mathrm{Cl} e(S)$ such that $\lambda(L(s) g)=0$ and $\lambda(g)=0$ for all $s \in S, g \in F$ with $0 \leqslant g \leqslant \xi_{T^{\prime}}$.

(f) For each $g \in F$ with $0 \leqslant g \leqslant \xi_{T^{\prime}}$, there is a mean $\lambda_{g} \in \mathrm{Cl} e(S)$ such that $\lambda_{g}(L(s) g)=0$ and $\lambda_{g}(g)=0$ for all $s \in S$.

Proof. (a) $\Leftrightarrow$ (b) by Theorem 3.1 (with $f=1-g$ ). (b) clearly implies (c). Assume (c), then for each $g \in F, 0 \leqslant g \leqslant \xi_{T^{\prime}}$, the set $K_{g}=\{m \in \mathrm{Cl} e(S): m(L(s) g)=0$ for all $s \in S\}$ is a nonempty weak* closed subset of the weak* compact set $\mathrm{Cl} e(S)$. Since $F$ is a lattice, if $g_{i} \in F, 0 \leqslant g_{i} \leqslant \xi_{T^{\prime}}, 1 \leqslant i \leqslant n$, then $g_{0}=\sup \left\{g_{i}: 1 \leqslant i \leqslant n\right\}$ $\in F$ and $0 \leqslant g_{0} \leqslant \xi_{T^{\prime}}$. Moreover $\bigcap\left\{K_{g_{i}}: 1 \leqslant i \leqslant n\right\} \supset K_{g_{0}}$. Hence $\left\{K_{g}: g \in F\right.$, $\left.0 \leqslant g \leqslant \xi_{T}\right\}$ has the finite intersection property which implies $\cap\left\{K_{g}: g \in F\right.$, $\left.0 \leqslant g \leqslant \xi_{T^{\prime}}\right\} \neq \varnothing$ and (c) implies (b). Also if $T$ is left $F$-thick, take $\lambda$ as in (b) and $s_{\alpha} \in S$ such that $e\left(s_{\alpha}\right) \rightarrow \lambda$ in $S_{1}$. Then $R\left(s_{\alpha}\right) g \rightarrow 0$ pointwise on $S$ and $T$ is right $F$-stationary. Conversely, if $T$ is right $F$-stationary, then $R\left(s_{\alpha}\right) g \rightarrow 0$ pointwise on $S$. There is some subnet $s_{\beta}$ such that $e\left(s_{\beta}\right) \rightarrow \lambda_{g} \in \mathrm{Cl} e(S)$ and so for each $s \in S$,

$$
\lambda_{g}(L(s) g)=\lim _{\beta} e\left(s_{\beta}\right)(L(s) g)=\lim _{\beta} R\left(s_{\beta}\right) g(s)=0
$$

and $T$ is left $F$-thick. Thus (a), (b), (c) and (d) are all equivalent. The remaining part of the theorem follows from the fact that if $\lambda(L(s) g)=0$ for all $s \in S$, then $\lambda \odot \lambda$ satisfies $(\lambda \odot \lambda)(L(s) g)=0$ for all $s \in S$.

Remarks. There is a version of Theorem 3.4 in terms of the means $M(F)=$ $\mathrm{ClCo} e(S)$, the weak* closure of the convex hull of $e(S)$. More precisely, Theorem 3.4 remains valid if we replace $\mathrm{Cl} e(S)$ by $\mathrm{ClCo} e(S)$, the net $R\left(s_{\alpha}\right) g$ by a net of convex combinations in $\{R(s) g: s \in S\}$ and multiplicative left introvertedness by left introvertedness throughout (including the definition of right stationarity of $T$ ).

The above reformulation of left thickness (Theorem 3.4(b)) leads to the following Dixmier-Granirer type of criterion.

Let $H_{T}$ be the linear subspace of functions in $F$ of the form $\sum_{i=1}^{n} \alpha_{i} L\left(s_{i}\right) g_{i}, \alpha_{i}$ real, $s_{i} \in S, 0 \leqslant g_{i} \leqslant \xi_{T^{\prime}}, g_{i} \in F, 1 \leqslant i \leqslant n, n=1,2, \ldots\left(H_{T}\right.$ is the linear space generated by $O_{L}(T)=\left\{L(s) g: s \in S, g \in F, 0 \leqslant g \leqslant \xi_{T^{\prime}}\right\}$.) If $F$ is an algebra, we define $K_{T}$ as the ideal of all functions in $F$ of the form $\sum_{i=1}^{n} f_{i} L\left(s_{i}\right) g_{i}, f_{i}, g_{i} \in F$, $0 \leqslant g_{i} \leqslant \xi_{T^{\prime}}, s_{i} \in S, 1 \leqslant i \leqslant n, n=1,2, \ldots\left(K_{T}\right.$ is the ideal generated by $O_{L}(T)$.)

THEOREM 3.5. If $F$ is a lattice, then the following statements are equivalent:

(1) $T$ is left $F$-thick.

(2) $\sup \{h(s): s \in S\} \geqslant 0$ for all $h \in H_{T}$.

(3) $\inf \left\{\|1-h\|: h \in H_{T}\right\}=1$.

Each one implies

(4) $H_{T}$ is not (sup norm) dense in $F$.

If $S$ is a group, then all four are equivalent.

Proof. Using Theorem 3.1 and the arguments used in Granirer [6, Lemma 3, p. 99] we obtain the implications (1) $\Rightarrow(2) \Rightarrow(3) \Rightarrow(4)$. For $(3) \Rightarrow(1)$, we use Theorem 3.1 again and the arguments in Hewitt and Ross [8, Theorem 17.15, p. 235]. Finally 
if $S$ is a group, then (4) implies (1) by repeating the proof in Hewitt and Ross [8, Theorem 17.15 (iii) $\Rightarrow(\mathrm{i})$, p. 236] and noting that since $S$ is a group, if $f \in F$, $0 \leqslant f \leqslant \xi_{T^{\prime}}$, and $0 \leqslant h \leqslant L(s) f, s \in S$, then $h=L(s) g$ where $g=L\left(s^{-1}\right) h \in F$, and $0 \leqslant g \leqslant f$.

REMARK. If $S$ is only left cancellative, then (4) implies (1) provided $F=B(S)$ (see [8, Theorem 17.15 (iii) implies (i)]).

THEOREM 3.6. If $F$ is a norm closed subalgebra of $B(S)$, then the following statements are equivalent:

(1) $T$ is left $F$-thick.

(2) $\sup \{k(s): s \in S\} \geqslant 0$ for all $k \in K_{T}$.

(3) $\inf \left\{\|1-k\|: k \in K_{T}\right\}=1$.

(4) $K_{T}$ is not (sup norm) dense in $F$.

Proof. This follows from Theorem 3.1(c) and the arguments used in the proof of Granirer [6, Theorem 3, p. 99].

REMARK. We do not need $S$ to be a group nor lefft cancellation in $S$ to get the implication (4) implies (1) of Theorem 3.6.

\section{REFERENCES}

1. M. M. Day, Amenable semigroups, Illinois J. Math. 1 (1957), 509-544.

2. __ Semigroups and amenability, Proceedings, Wayne State University Symposium on Semigroups, edited by K. W. Folley, Academic Press, New York, 1969, pp. 5-54.

3. _ Lumpy subsets in left-amenable locally compact semigroups, Pacific J. Math. 62 (1976), 87-92.

4. __ Left lumpy to left thick, $A$ guided tour (to appear).

5. J. Dixmier, Les moyennes invariantes dans les semi-groupes et leurs applications, Acta Sci. Math. Szeged 12 (1950), 213-227.

6. E. Granirer, Extremely amenable semigroups. II, Math. Scand. 20 (1967), 93-113.

7. E. Granirer and A. Lau, A characterisation of locally compact amenable groups, Illinois J. Math. 15 (1971), 249-257. 1963.

8. E. Hewitt and K. A. Ross, Abstract harmonic analysis. I, Springer-Verlag, Berlin and New York,

9. H. D. Junghenn, Amenability of function spaces on thick subsemigroups, Proc. Amer. Math. Soc. 75 (1979), 37-41.

10. H. Kharaghani, Left thick subsets of a topological semigroup, Illinois J. Math. 22 (1978), 41-48.

11. T. Mitchell, Constant functions and left invariant means on semigroups, Trans. Amer. Math. Soc. 119 (1965), 244-261.

12. __ Function algebras, means and fixed points, Trans. Amer. Math. Soc. 130 (1968), 117-126.

13. C. Wilde and K. G. Witz, Invariant means and the Stone-Čech compactification, Pacific J. Math. 21 (1967), 577-586.

14. James C. S. Wong, Topologically stationary locally compact groups and amenability, Trans. Amer. Math. Soc. 144 (1969), 351-363.

15. ___ Amenability and substantial semigroups, Canad. Math. Bull. 19 (1976), 231-234.

16. A characterisation of topological left thick subsets in locally compact left amenable semigroups, Pacific J. Math. 62 (1976), 295-303.

17. __ On topological analogues of left thick subsets in semigroups, Pacific J. Math. 83 (1979), $571-585$.

Department of Mathematics and Statistics, University of Calgary, Calgary, Alberta, CANADA T2N 1N4 